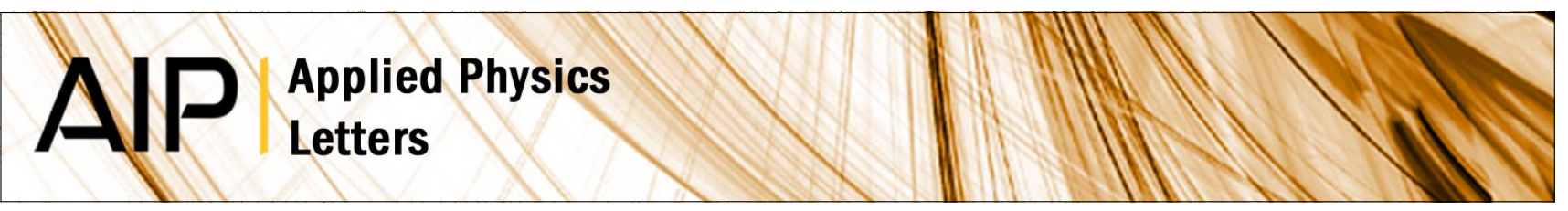

\title{
Direct mapping of surface plasmon dispersion using imaging scatterometry
}

Thomas J. Constant, Alastair P. Hibbins, Alfred J. Lethbridge, J. Roy Sambles, Edmund K. Stone et al.

Citation: Appl. Phys. Lett. 102, 251107 (2013); doi: 10.1063/1.4812482

View online: http://dx.doi.org/10.1063/1.4812482

View Table of Contents: http://apl.aip.org/resource/1/APPLAB/v102/i25

Published by the AIP Publishing LLC.

Additional information on Appl. Phys. Lett.

Journal Homepage: http://apl.aip.org/

Journal Information: http://apl.aip.org/about/about_the_journal

Top downloads: http://apl.aip.org/features/most_downloaded

Information for Authors: http://apl.aip.org/authors

\section{ADVERTISEMENT}
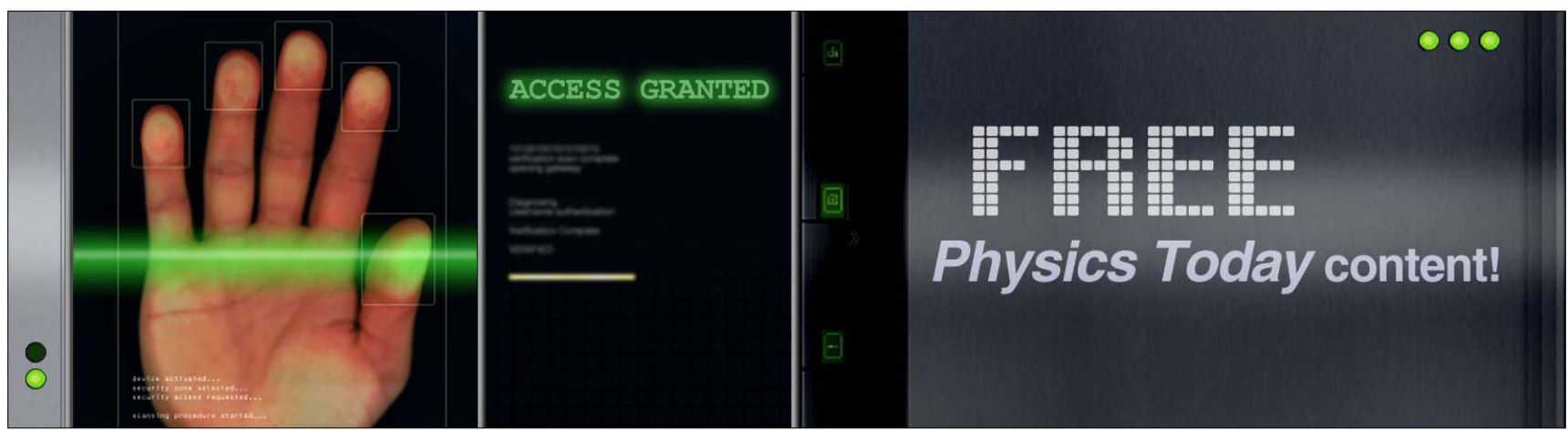


\title{
Direct mapping of surface plasmon dispersion using imaging scatterometry
}

\author{
Thomas J. Constant, ${ }^{\text {a) }}$ Alastair P. Hibbins, Alfred J. Lethbridge, J. Roy Sambles, \\ Edmund K. Stone, and Pete Vukusic \\ Department of Physics and Astronomy, University of Exeter, Stocker Road, Exeter, Devon EX4 4QL, \\ United Kingdom
}

(Received 16 May 2013; accepted 11 June 2013; published online 25 June 2013)

\begin{abstract}
The iso-frequency contours of diffracted surface plasmons on metallic bigratings have been directly recorded using imaging scatterometry. Metallic rectangular bigratings, formed of two "crossed" surface relief gratings, are used to demonstrate this measurement technique. By deepening one of the constituent gratings, control of the surface plasmon dispersion anisotropy is shown in the recorded iso-frequency maps. Collating the iso-frequency contours over a range of wavelengths from $500 \mathrm{~nm}$ to $700 \mathrm{~nm}$ leads to a three-dimensional map of the surface plasmon dispersion. (C) 2013 AIP Publishing LLC. [http://dx.doi.org/10.1063/1.4812482]
\end{abstract}

The optical response of structured metallic surfaces is an active and extremely popular area of electromagnetic research. ${ }^{1}$ Illuminating metal surfaces structured on the order of, or far below, the wavelength of the incident light leads to some interesting optical effects, many of which are mediated by the excitation of trapped electromagnetic surface waves coupled to the conduction-electron plasma of metals. The resulting surface wave quanta are named surface plasmons (SPs).

Once an incident field has been trapped at the surface, there exists the potential to funnel the wave along the surface, to focus it, ${ }^{2-4}$ to collimate it, ${ }^{5,6}$ or even to cause its negative refraction, ${ }^{7}$ all by suitable design of the surface structure. Such metamaterials form part of a tool-kit for surface plasmon optics and provide a possible intermediary between light and electronics that is increasingly in demand. ${ }^{8}$

A useful measurement for understanding SP optics is the mapping of the SP's iso-frequency contours. Iso-frequency contours (IFCs) are maps of the allowed momentum states of SPs in reciprocal space at a fixed frequency. On such maps, the direction of the SP group velocity, $\mathbf{v}_{g}=\nabla_{k} \omega(\mathbf{k})$, lies normal to the SP contours, showing in which direction a surface wave with a given wavevector will propagate power. The SP dispersion relation, $\omega(\mathbf{k})$, can be mapped by collating together multiple iso-frequency contours for a range of frequencies, $\omega$. This provides a more complete understanding of surface-wave optics and their ability to influence the propagation of SPs.

In order to excite SPs using free-space light, a coupling mechanism is required to match the wavevector (equivalently momentum) of the light field to that of the surface modes. One of the most common of these methods is the use of a diffraction grating. Various methods exist for mapping the IFCs and the dispersion relation for such a diffracting plasmonic system. The traditional method is to measure the reflection or transmission of light from a sample as a function of polar angle for multiple azimuthal angles. ${ }^{9}$ The angle of the reflectivity anomaly associated with SP excitation may then be used to map the mode position in momentum space. However, when the plane of incidence lies tangential to a SP contour the recorded reflectivity modes can become

${ }^{\text {a)} E l e c t r o n i c ~ m a i l: ~ t . j . c o n s t a n t @ e x . a c . u k ~}$ broad, and determining the anomaly's position can be challenging.

Another solution is to directly map the dispersion by exciting SPs on a metal surface in all planes simultaneously and to simply record the resulting transmission image using a camera. This provides high resolution, raster-free maps of $k$-space. This has been achieved recently using the direct transmission for thin grating samples, ${ }^{10}$ with leaky radiation setups, ${ }^{6,11}$ and using wide-field leakage of SP-coupled florescence techniques. ${ }^{12-14}$ Direct imaging of IFCs has also been achieved for plasmonic samples with defects. ${ }^{15}$

We present here a method to map the surface plasmon IFCs using instead the reflection from a plasmonic surface. By adapting an imaging scatterometer, ${ }^{16-18}$ our method allows the mapping of the diffracted IFCs without the addition of fluorophores or any other modifications to the sample surface. Since the technique measures the reflection from a sample surface, the sample itself may be optically thick, entirely free from defects, and the illuminating light source can be one of low intensity, such as a filament lamp. The use of such a low intensity source, as opposed to a laser, enables the mapping of the IFCs for a range of frequencies by employing different colour filters. The apparatus is relativity simple, inexpensive and allows quantitative comparison between the experimentally measured IFCs and the associated model predictions.

To demonstrate the technique, the IFCs for two SPsupporting diffraction gratings are mapped. The first is a "shallow" ( $\approx 60 \mathrm{~nm}$ deep) rectangular bigrating, and the second grating is designed to be identical save for the grating depth in one direction, which is made much deeper $(\approx 120 \mathrm{~nm})$. By deepening one grating, the SP IFCs' shape may be controlled. These IFCs are recorded for a range of wavelengths that when collated together produce a 3D map of the SP dispersion.

Scatterometry has been used previously to record the scattering patterns from samples found in Natural Photonics, such as the naturally diffuse scattering of the Chrysochroa fulgidissima beetle ${ }^{17}$ or the Eupholus magnificus beetle. ${ }^{19}$ It has also been used to show the hexagonal shaped Brillouin zone of a diamond-type lattice found in the Entimus imperialis weevil. ${ }^{20}$ 
In our work, the previously reported scatterometry experimental arrangement ${ }^{16,18}$ has been modified by the addition of spectral filters to limit the image acquired to a narrow wavelength band. By applying a simple geometric conversion to the image, the scatterometer provides a map of the coupled SP IFCs in momentum-space over the entire incident light circle.

A schematic of the scatterometry arrangement is shown in Fig. 1(a). White light is directed through a collimating lens, L1, and a linear polariser that allows the investigation of the polarisation sensitivity of the acquired image. For the results presented here, the polariser is set so that the electric field vector lies parallel to the $\mathbf{k}_{g x}$ direction. It is then focussed through an alignment pinhole and the beam is reflected via the beam-splitter (BS) on to an ellipsoidal mirror with an eccentricity of 0.833 . The mirror, M1, focusses the light onto the sample positioned at $\mathrm{G}$.

If aligned precisely, the cone of light focussed by the mirror will include light from all azimuthal, $\phi$, angles and a range of polar angles from $\theta \approx 5^{\circ}$ to $\theta \approx 90^{\circ}$. The lower limit of $\theta$ is determined by the shadow cast by the sample, meaning that the sample size (in this case $\approx 2 \mathrm{~mm}^{2}$ ) places a constraint on the lower limit of $k$ measured.

The reflected light from the sample is then collected by the same ellipsoidal mirror, M1, and is focussed through a second alignment pinhole, P2, positioned at the mirror's secondary focal point. This light is then collimated so that the polar angle $\theta$ is approximately linearly proportional to the radial axis, $r$, of the obtained image. The beam is finally passed through a spectral filter and imaged using a CCD camera. The acquired image is a directly mapped reflectivity plot of all polar and azimuthal angles, $R(\theta, \phi)$, with a resolution determined by the pixel size and density of the CCD. Using a range of spectral filters positioned at $\mathrm{F}$, the wavelength and so the fixed frequency of the map can be selected. The filters used were $500 \pm 5 \mathrm{~nm}, 550 \pm 5 \mathrm{~nm}, 580 \pm 5 \mathrm{~nm}, 600 \pm 5 \mathrm{~nm}$, $650 \pm 5 \mathrm{~nm}$, and $700 \pm 5 \mathrm{~nm}$. The reflectivity of the sample for a narrow wavelength band over the range $0^{\circ}<\phi<360^{\circ}$ and $5^{\circ}<\theta<90^{\circ}$ is thus recorded in a single image by the CCD.

Fig. 1(c) shows a diagram of the allowed SP momentum states in $k$-space for a rectangular lattice. The momentum of a SP on a flat interface (red line) is greater than that of grazing free-space light (green line), and so the surface wave cannot couple to optical fields and is non-radiative; the allowed SP momentum states of $k_{S P}$ lie at greater values of wavevector than that of incident light $\left(k_{0}\right)$ in all directions along the surface. Bragg scattered SP and light circles for a rectangular lattice are also shown in Figure 1(c) as black and blue circles, respectively, scattered by integer values of the lattice vectors. By adding a periodic structure to the surface (as seen in the scanning electron micrograph of the sample, inset in Fig. 1(b)), SPs may now scatter into the radiative light circle and may then couple to free space light, as shown in the figure as black circles lying inside the green zero-order light circle.

At the values of $\theta$ and $\phi$ which match the momentum of such a SP, a reflectivity anomaly will be found in the acquired image. These reflectivity anomalies, to a first approximation, map the position of the SP IFCs over the large range of $\theta$ and $\phi$ available to this experiment. These SP

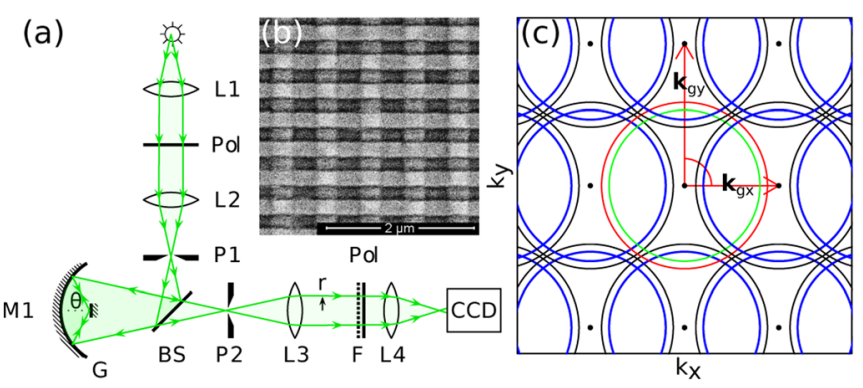

FIG. 1. (a) The experimental arrangement of the imaging scatterometer. (b) A scanning electron micrograph of a silver rectangular bigrating used in this work, scale bar: $2 \mu \mathrm{m}$. (c) A reciprocal-space map for diffracted light circles (blue/green lines) and SP modes (black/red lines) for the grating shown in (b).

contours mapped in momentum-space are the same contours that are measured using imaging scatterometry.

To obtain a map of $k$-space from the scatterograms, two image adjustments are required. The first corrects the aberration of the ellipsoidal mirror to obtain an image whose radial axis is linearly scaled with respect to the polar angle, $\theta$. This small correction is detailed in Ref. 16. The second adjustment scales the radial axis of the image to be proportional to the in-plane momentum such that the reflectivity plot, $R(\theta, \phi)$, becomes

$$
R(\theta, \phi) \rightarrow R\left(k_{0} \sin \theta, \phi\right) \equiv R\left(k_{x}, k_{y}\right),
$$

where $k_{0} \sin \theta$ is the in-plane momentum for a photon in the plane of incidence, at an azimuthal angle $\phi$. Due to the $\sin \theta$ dependence of the adjusted radial axis, a large range of $k$ space is mapped for a relativity small polar angle range. For example, if the angle of illumination is only $\theta=60^{\circ}$, over $80 \%$ of the light cone is mapped.

The diagram in Fig. 1(c) shows the simple picture of SP contours as circles centred about their respective lattice points. However, interaction between Bragg scattered SPs deforms these contours. When a propagating SP meets a scattered counter-propagating SP of equal wavevector and energy, two possible SP standing-waves may form. ${ }^{21}$ On a grating, these SP standing-waves may differ in energy due to the different arrangements of the induced charge-density across the surface, forming a band-gap in the SP dispersion.

The sizes of the SP band-gaps are proportional to the diffraction efficiency required to couple two counterpropagating SPs together to form the required SP standing waves. They are therefore proportional to the amplitude of the grating harmonics responsible for this coupling. ${ }^{21} \mathrm{By}$ simply altering a grating's amplitude, the size of SP bandgaps may be controlled.

On a rectangular bigrating there are two constituent lamellar gratings oriented orthogonally to one another. It will subsequently be shown that SP band-gaps in one direction may be controlled by deepening the appropriate grating, leaving the band-gaps that form in the orthogonal direction (along the other constituent grating) largely unaffected. This allows the design of the SP IFCs, where the SP dispersion varies largely depending on the direction in which the SP travels along the grating. In this work, the short-pitch grating has been deepened, and the effect on the SP IFCs is observed by using imaging scatterometry. 
Two bigratings were produced for this experiment, with the aim that they be identical save for the depth of the shorter-pitch grating, $d_{y}$. The two gratings are manufactured at the same time via electron beam lithography, using the same electron dose to expose the pattern. The target long pitch was $\lambda_{g x}=600 \mathrm{~nm}$ and for the short pitch $\lambda_{g y}=400 \mathrm{~nm}$. The target depth of the long pitch for both gratings was $d_{x}=35 \mathrm{~nm}$, which is achieved by reactive ion etching the masters in the same etching exposure run. The second shortpitch grating was then added by spinning a second resist layer over the master and exposing the short-pitch grating pattern via electron beam lithography. The depth of this second shorter-pitch grating was varied between samples, again using reactive ion etching. For the "shallow" bigrating, the target depth of $d_{y}=60 \mathrm{~nm}$ was used; for the "deep" bigrating the target depth was $d_{y}=120 \mathrm{~nm}$. The final parameters of the sample, measured from the scanning electron micrograph shown in Fig. 1(b), were $\lambda_{g x}=592 \pm 10 \mathrm{~nm}$ and $\lambda_{g y}=$ $395 \pm 9 \mathrm{~nm}$ with the duty cycle (the ratio between the length of the lamellar grating peaks and the pitch) of the grating in the $x$-direction measured as $\Gamma_{x}=0.45$ and in the $y$-direction as $\Gamma_{y}=0.54$.

Figure 2 shows the experimentally mapped isofrequency contours for the two rectangular bigratings at a wavelength of $\lambda_{0}=700 \mathrm{~nm}$. Figure 2(a) shows two SP contours mapped from the shallow grating as dark bands of reflectivity closely following the $\pm 1 \mathbf{k}_{g x}$ scattered diffraction circles (blue lines). These SP contours exhibit a small degree of anisotropy with respect to their associated diffracted light circle, with the SP contour lying closer to the diffracted light lines at $k_{y}=0$ than elsewhere along the contour. This is due to the $( \pm 1,0)$ scattered SPs interacting and forming band gaps with the $( \pm 1, \pm 1)$ or $(\mp 1, \pm 1)$ SPs. Separated by a single scattering vector, $\mathbf{k}_{g y}$, the increase in the diffraction efficiency of the $k_{g y}$ grating harmonic (by deepening the grating in that direction) will be the dominant mechanism for the changing of this band-gap's size. This interaction is strong as it only requires a single scattering event of $\pm 1 \mathbf{k}_{g y}$ to couple the SPs together. The strength of this interaction causes the perturbation in the value for $k_{S P}$ from a perfect circle centred around the $( \pm 1,0)$ lattice point.

By increasing the diffractive coupling between SPs by deepening the grating in the $y$-direction, the $( \pm 1,0)$ SPs will
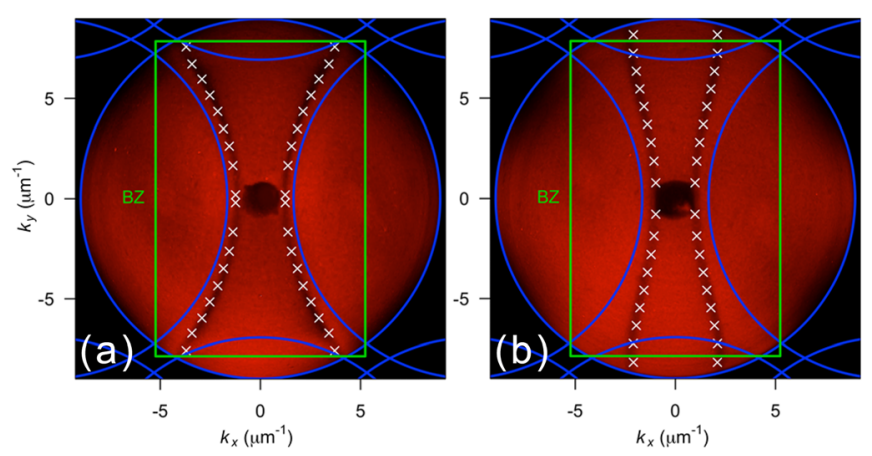

FIG. 2. Mapped iso-frequency contours at a fixed wavelength of $\lambda_{0}=$ $700 \mathrm{~nm}$ for (a) the shallow bigrating $\left(d_{y} \approx 60 \mathrm{~nm}\right)$ and $(\mathrm{b})$ the deep bigrating $\left(d_{y} \approx 120 \mathrm{~nm}\right)$. The blue circles indicate the diffraction circles for grazing light, and the white points show mode position for the modelled IFCs. The green line indicates the BZ boundary. form larger band-gaps at the 1st Brillouin Zone (BZ) boundary in the $k_{y}$ direction (at which $\mathbf{v}_{g y} \rightarrow 0$ ). The effect of this is to "flatten" the band along the $k_{y}$ axis. By deepening the short-pitch grating to $120 \mathrm{~nm}$ this anisotropy is increased. This is seen clearly in the experimental results in Figure 2(b) where the $( \pm 1,0)$ scattered SPs' interaction with the $( \pm 1, \pm 1)$ and $(\mp 1, \pm 1)$ scattered SPs have served to flatten the SP contours, with the IFCs near the BZ boundary lying further from their associated diffraction circles.

The theoretically calculated SP mode positions in these IFCs are overlaid on to the scatterograms in Fig. 2. These were calculated using the Chandezon method, ${ }^{22,23}$ with the grating surface represented by a Fourier series with the Fourier amplitudes, $a_{n}$, for the $n t h$ harmonic determined by, $a_{n}=(4 A / n \pi) \sin n \pi \Gamma$. The fundamental amplitude in the $x$-direction for both gratings was set as $A=35 \mathrm{~nm}$, and the amplitude in the $y$-direction set to $A=37 \mathrm{~nm}$ for the "shallow" grating and $A=60 \mathrm{~nm}$ for the deep grating. Values for $\Gamma$ were set equal to the measured values from the scanning electron micrograph (Fig. 1(b)). The silver permittivity was fitted to the data and found to be $\varepsilon=-23+0.6 i$, which is within $5 \%$ of typical values found in literature. ${ }^{24}$ The dominant scattering observed in this experiment was due to the $\pm 1 \mathbf{k}_{g y}$ component. Truncating the Fourier series to the first four components was sufficient to obtain good agreement between the model and the data.

The dispersion of the SPs on these gratings was then mapped by changing the spectral filter for a range of wavelengths. Figure 3 shows the dispersion for the SPs on the deeper grating. At $\lambda_{0}=600 \mathrm{~nm}$, the scatterogram is taken at a frequency for which part of the SP IFC lies close to the forbidden frequency range in the band-gap, and so appears as a weak reflection feature. Due to the SP anisotropy, the SP's group velocity (lying normal to the contours) for a range of momenta are equal and opposite as they meet at $k_{x}=0$, forming standing-waves and, as a result, a band-gap. If a scatterogram is taken at a frequency which lies inside the forbidden propagation frequency range, no SP contours will be observed. Figure 3 also shows that the curvature of the SP contours decreases with frequency until at $\lambda_{0}=500 \mathrm{~nm}$, the IFCs are almost perfectly flat, with the group velocity having no component in the $\mathbf{k}_{y}$ direction. The ability for such gratings to direct SPs solely in the $\mathbf{k}_{x}$ direction could form the basis of a SP collimation device, with the SP group velocity in a single direction, irrespective of the in-plane momentum of the coupling light.

Notice in these scatterograms, just as in Figure 2, the $(0, \pm 1)$ SP contours are not observed due to the incident light polarisation state chosen for these images, which was selected to highlight the $( \pm 1,0)$ SP IFCs. The $( \pm 1, \pm 1)$, $(\mp 1, \pm 1)$ scattered SP IFCs are not observed since for this particular grating they require multiple scattering events to Bragg scatter into the zero-order cone-an inherently weak process.

In conclusion, the iso-frequency contours of surface plasmons on two rectangular bigratings have been mapped using imaging scatterometry. This demonstrates an original and time-saving technique for mapping these contours which has not been reported previously. The experimental arrangement presented is an imaging scatterometer, adapted for use 

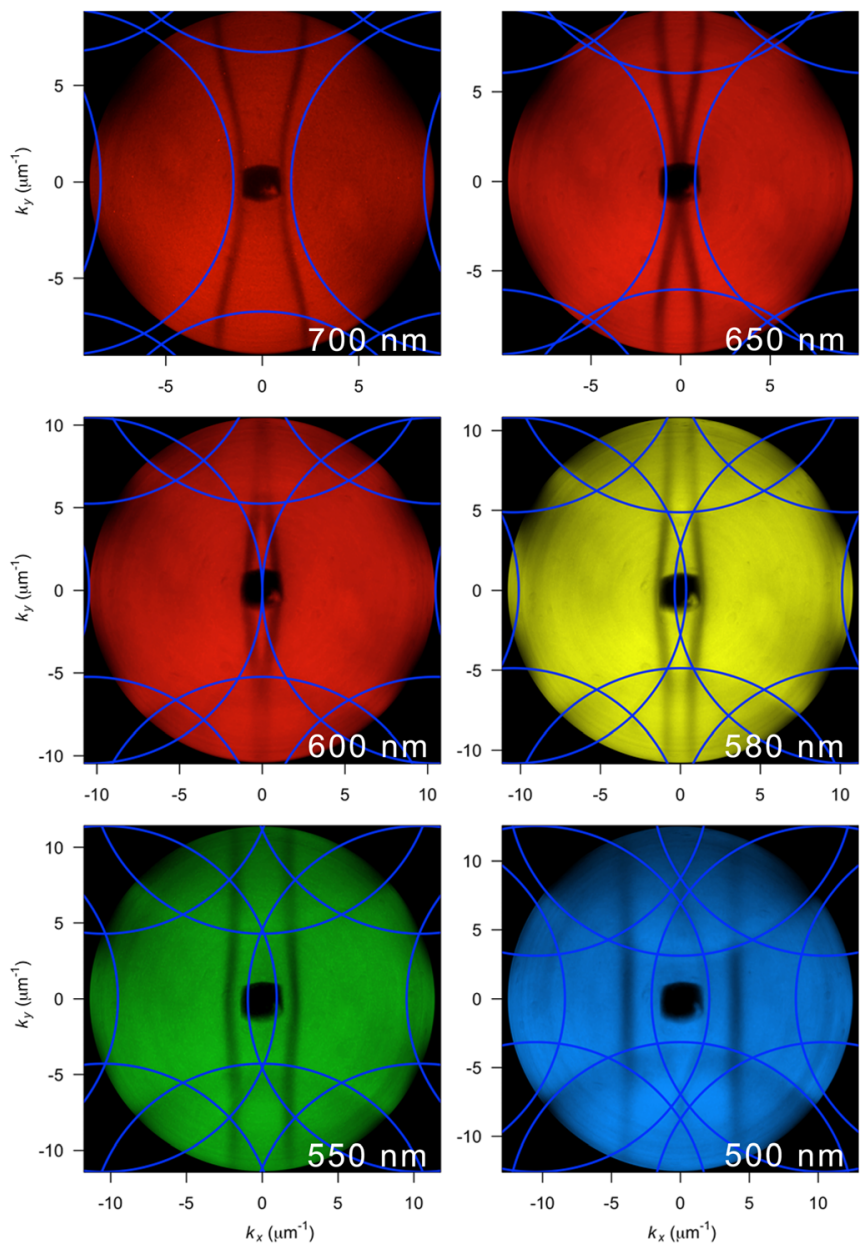

FIG. 3. The collated IFCs for SPs on the deep bigrating. The illuminating wavelength is indicated on each scatterogram. Blue lines indicate the momentum states for grazing diffracted orders of light (light circles).

as an IFC mapping device for SPs. Using this technique, the IFCs for SPs supported on two example gratings with rectangular symmetry have been mapped, and the manipulation of the IFC shape by changing groove depths of a constituent grating has been shown experimentally.

The use of filters in our experimental arrangement limits the spectral resolution to steps of $\pm 50 \pm 5 \mathrm{~nm}$. An obvious improvement to this experimental arrangement is the use of a white light source and a monochromator to scan through wavelengths over a larger spectral range with higher spectral resolution. The same improvement could be made with any other tunable light source.

The SP dispersion measured here demonstrates how SP IFC shape may by manipulated on a rectangular bigrating by deepening one of the constituent grating grooves, and the experimentally mapped IFCs show good agreement with model predictions. In the results presented here, the IFC shape on a rectangular bigrating was modified to such a degree that for an illumination wavelength of $\lambda_{0}=500 \mathrm{~nm}$, the group velocity of the excited SPs is in a single direction for any incident plane of coupled free-space light. This mechanism of using an orthogonal grating to affect the dispersion of SPs could form the basis of a SP collimation device, with SPs on a grating constrained to travel parallel to one another in the $\mathbf{k}_{x}$ direction.

The authors would like to thank David Anderson for assistance in the electron beam lithography and Ian Hooper for helpful discussions. Thanks also to Doekele Stavenga and Hein Leertouwer for providing the original scatterometer. Sponsorship for this work was received from the EPSRC (Grant No. EP/G022550/1), HP Labs Bristol, and the USAF (Award No. FA9550-10-1-0020).

${ }^{1}$ W. L. Barnes, A. Dereux, and T. W. Ebbesen, Nature (London) 424, 824 (2003).

${ }^{2}$ H. L. Offerhaus, B. van den Bergen, M. Escalante, F. B. Segerink, J. P. Korterik, and N. F. van Hulst, Nano Lett. 5, 2144 (2005).

${ }^{3}$ Y. Ogawa, Y. Omura, S. Member, and Y. Iida, J. Lightwave Technol. 23, 4374 (2005).

${ }^{4}$ L. Yin, V. Vlasko-Vlasov, J. Pearson, J. Hiller, J. Hua, U. Welp, D. E. Brown, and C. W. Kimball, Nano Lett. 5, 1399 (2005).

${ }^{5}$ S. Kim, T. Kim, S. Oh, J. Kim, H. Park, and C. Kee, Phys. Rev. B 83, 165109 (2011).

${ }^{6}$ B. Stein, E. Devaux, C. Genet, and T. Ebbesen, Opt. Lett. 37, 1916 (2012). ${ }^{7}$ C. Luo, S. Johnson, J. Joannopoulos, and J. Pendry, Phys. Rev. B 65, 201104 (2002).

${ }^{8}$ E. Ozbay, Science 311, 189 (2006).

${ }^{9}$ R. A. Watts, J. Harris, A. Hibbins, T. Preist, and J. R. Sambles, J. Mod. Opt. 43, 1351 (1996).

${ }^{10}$ A. Giannattasio and W. Barnes, Opt. Express 13, 428 (2005).

${ }^{11}$ A. Drezet, A. Hohenau, D. Koller, A. Stepanov, H. Ditlbacher, B. Steinberger, F. R. Aussenegg, A. Leitner, and J. R. Krenn, Mater. Sci. Eng. B 149, 220 (2008).

${ }^{12}$ S. Frisbie, C. Chesnutt, M. Holtz, A. Krishnan, L. de Peralta, and A. Bernussi, IEEE Photon. J. 1, 153 (2009).

${ }^{13}$ C. J. Regan, A. Krishnan, R. Lopez-Boada, L. G. de Peralta, and A. A. Bernussi, Appl. Phys. Lett. 98, 151113 (2011).

${ }^{14}$ C. J. Regan, L. G. de Peralta, and A. A. Bernussi, J. Appl. Phys. 111, 073105 (2012).

${ }^{15}$ L. Shi, H. Yin, X. Zhu, X. Liu, and J. Zi, Appl. Phys. Lett. 97, 251111 (2010).

${ }^{16}$ D. G. Stavenga, H. L. Leertouwer, P. Pirih, and M. F. Wehling, Opt. Express 17, 193 (2009).

${ }^{17}$ D. G. Stavenga, B. D. Wilts, H. L. Leertouwer, and T. Hariyama, Philos. Trans. R. Soc. B 366, 709 (2011).

${ }^{18}$ P. Vukusic and D. G. Stavenga, J. R. Soc. Interface 6(Suppl 2), S133 (2009).

${ }^{19}$ C. Pouya, D. G. Stavenga, and P. Vukusic, Opt. Express 19, 11355 (2011).

${ }^{20}$ B. D. Wilts, K. Michielsen, H. De Raedt, and D. G. Stavenga, J. R. Soc. Interface 9, 1609 (2012).

${ }^{21}$ W. L. Barnes, T. W. Preist, S. C. Kitson, and J. R. Sambles, Phys. Rev. B 54, 6227 (1996).

${ }^{22}$ J. B. Harris, T. W. Preist, J. R. Sambles, R. N. Thorpe, and R. A. Watts, J. Opt. Soc. Am. A 13, 2041 (1996).

${ }^{23}$ J. Chandezon, D. Maystre, and G. Raoult, J. Opt. (Paris) 11, 235 (1980).

${ }^{24}$ D. J. Nash and J. R. Sambles, J. Mod. Opt. 43, 81 (1996). 